\title{
Dysphagia Secondary to Spirocerca lupi in a German Shepherd Dog
}

\author{
Carlos Arturo Rodríguez-Alarcón, Andrea López-Aguilar, Federico Pérez-Casio, Ramón Rivera-Barreno, Oliver \\ Castillo-Luna \& Diana Marcela Beristain-Ruiz
}

\begin{abstract}
Background: Spirocerca lupi is a nematode that causes nodules in the esophagus and aortic aneurysms in dogs. Animals are infected by eating intermediate host (beetle) containing infective larvae or consuming viscera containing parasitic cysts from paratenic hosts. Dogs commonly have a subclinical presentation, but the most common signs are vomiting and regurgitation. Visualizing nodules or neoplastic masses via esophagoscopy and the presence of $S$. lupi eggs in feces are the best diagnostic methods. Milbemycin oxime is effective as a treatment for $S$. lupi. The objective of this study was to describe the diagnostic and medical management of a patient with dysphagia secondary to esophageal $S$. lupi nodules.

Case: A 7-year-old male German shepherd dog was referred for endoscopic examination. The animal presented with chronic severe dysphagia and weight loss with a presumptive diagnosis of an esophageal foreign body. At palpation of the neck, the dog was painful just dorsal to the cricoid cartilage and had submandibular lymphadenomegaly. The animal also exhibited a dropped jaw which could be returned to its normal position by manipulation. Radiographic examination demonstrated a radiopaque image compatible with a mass in the cranial esophagus just caudal to the upper esophageal sphincter. A fluoroscopy study was performed and confirmed the presence of a mass apparently within the esophageal wall preventing the passage of food. Additionally, in this case, small amounts of contrast medium were visualized passing into the dog trachea. The animal was hospitalized exhibiting odynophagia, dysphagia and regurgitation. Blood analysis revealed a hypochromic normocytic anemia, severe thrombocytopenia and hipoalbuminemia. A commercial ELISA test for Ehrlichia spp. and Anaplasma spp. was performed (SNAP 4Dx plus, IDEXX®). The test was positive for Ehrlichia spp. and doxycycline treatment was started. Fluoroscopy was performed using iodinated contrast, and we observed a soft tissue density structure at the ventral wall of the esophagus that was not permitting the passage of the contrast through the upper esophageal sphincter. One week after doxycycline treatment the animal showed a improvement, with appetite and increased weight. In addition, the dropping jaw was no longer evident, but, the dysphagia continued. Laboratory analyses were repeated, and values were within the normal ranges. Esophagoscopy was performed which allowed us to observe a mass suggestive of a nodule secondary to $S$. lupi. This nodule was found in the cranial esophagus and could not be biopsied. However S. lupi eggs were observed in the fecal study. The patient was treated with milbemycin oxime to which the patient responded favorably, regaining the ability to swallow and gaining weight.

Discussion: Spirocercosis is a parasitic disease with a worldwide distribution which occurs mainly in warm climates as in the present case. Dogs often have a subclinical presentation; however when present, the most common clinical signs are regurgitation and vomiting. In the present case the patient had severe chronic dysphagia as the principal sign, which makes this case atypical. Dysphagia occurred in this case because a nodule was formed in the cranial esophagus, which is also rare. This location made it impossible to take endoscopic biopsies, as described by other authors. However, the diagnosis was made by identifying microscopic parasites eggs in the stool test. In addition, dog improved with medical treatment with milbemycin oxime, at the present, no longer shows dysphagia, odynophagia or coughs and has improved their body condition. This is the first official report of the presence of canine Spirocerca lupi in Ciudad Juarez, Mexico. Finally, dysphagia is an uncommon sign in dogs with spirocercosis; however, esophageal nodules secondary to S. lupi should be considered as a cause of this sign.
\end{abstract}

Keywords: endoscopy, milbemycin oxime, Spirocerca lupi, nodule, dysphagia, dog. 


\section{INTRODUCTION}

Spirocercosis is a parasitic disease characterized by the presence of nodules in the esophagus, aorta and stomach [21]. Generally, causes a subclinical presentation, but, fatal lesions may occur due to aortic aneurysm or rupture of blood vessels [3]. The most common signs are vomiting, regurgitation and emaciation. To a minor degree, dyspnea, dysphagia, salivation, odynophagia, aspiration pneumonia and weight loss can be observed $[3,13]$. Previously, the nodules were referred to as granulomas, but histologically this is inappropriate [22]. Animals are infected by ingestion of beetles containing Spirocerca lupi larvae or from consumption of a variety of paratenic hosts [22]. The larvae penetrate the stomach wall and migrate through the arteries, and they arrive at the thoracic aorta from which they migrate to the esophagus. In the esophagus, the parasite reaches its adult stage and produces eggs that are eliminated in the feces [3]. Radiographic studies may be suggestive of $S$. lupi infection if they demonstrate mass in the esophagus. However, the definitive diagnosis is made by the presence of $S$. lupi eggs in the feces $[13,24]$. Additionally, with esophagoscopy we can observe nodules and esophageal damage and obtain biopsies $[1,3,13]$. Early to mature benign nodules are smooth, round and sessile that protrudes into the esophageal. Conversely, neoplastic nodules generally have a roughened, ulcerated and necrotic surface that should be biopsied [3]. Milbemycin oxime, have been evaluated as a treatment for $S$. lupi $[8,17]$. The aim of this study was to describe the diagnostic and medical management of a patient with severe dysphagia secondary to an $S$. lupi esophageal nodule.

\section{CASE}

A 7-year-old male German shepherd dog with history of cough and severe chronic dysphagia was referred for esophagoscopy. According to the owner, the cough had been present for two months and was exacerbated by food intake, and the dog also had sporadic painful swallowing and regurgitation. The referring veterinary clinic was suspicious of an esophageal foreign body.

Physical examination showed a body condition score of $2 / 5(22 \mathrm{~kg})$, according to the scale of Laflamme in 1997 [10]. The dog was painful on palpation of the cranial neck region just dorsal to the cricoid cartilage and had right submandibular lymphadenomegaly.
The dog also exhibited a dropped jaw which could be returned to its normal position by manipulation.

Plain radiographs of the cervical esophagus and thorax were taken. Gas in the cranial esophagus and the presence of a radiopaque structure with soft tissue density dorsal to the cricoid cartilage were observed on the lateral cervical radiograph (Figure 1). On the thoracic radiographs only a bronchial pattern was found.

The patient was hospitalized and administered meloxicam (Mobicox $\left.{ }^{\circledR}\right)^{1}$ at $0.2 \mathrm{mg} / \mathrm{kg}$ IM SID for analgesia and ranitidine (Ranulin $\left.{ }^{2}\right)^{2}$ at $2 \mathrm{mg} / \mathrm{kg} \mathrm{SC}$ $\mathrm{BID}$ for its prokinetic action and protective effects on the gastric mucosa. The patient was also started on intravenous fluid therapy with Hartmann's solution (Solucion HT PISA®) ${ }^{2}$ at the maintenance dose. During hospitalization the dog exhibited odynophagia, dysphagia and regurgitation.

Blood cell analysis revealed a hypochromic normocytic anemia and severe thrombocytopenia ( $55 \mathrm{x}$ $10^{9} / \mathrm{L}$, reference value $\left.160-465 \times 10^{9} / \mathrm{L}\right)$. Blood chemistry showed a urea concentration of $13.67 \mathrm{mmol} / \mathrm{L}$ (3.5-6.0 mmol/L), creatinine of $144 \mathrm{mmol} / \mathrm{L} \mathrm{(20-110}$ $\mathrm{mmol} / \mathrm{L})$ and hypoalbuminemia at $21.24 \mathrm{~g} / \mathrm{L}(26-35$ $\mathrm{g} / \mathrm{L})$. The urinalysis demonstrated hyperesthenuria. Because anemia and thrombocytopenia were found, a commercial ELISA test for Ehrlichia and Anaplasma was performed (SNAP 4Dx plus, IDEXX®) ${ }^{3}$. The test was positive for Ehrlichia, doxycycline (Vibramicina $\left.{ }^{4}\right)^{4}$ treatment was started $(10 \mathrm{mg} / \mathrm{kg}$ PO SID for 30 days) and the endoscopic procedure was postponed. However, fluoroscopy was performed using iodinated contrast administered within food, and we observed a soft tissue density structure at the ventral wall of the esophagus that was not permitting the passage of the contrast through the upper esophageal sphincter. In addition, the contrast fluid was observed leaking into the trachea (Figure 2).

The dog was fed in the high position as used in patients with megaesophagus. No regurgitation was observed and its appetite was increased in this position, but the dysphagia continued. At this point, the presumptive diagnosis for the dog was canine polymyositis secondary to ehrlichiosis as reported by Evans et al. [6]. This possibly explained the dropping jaw and the dysphagia due to inflammatory myositis of the masticatory muscles affecting muscles innervated by the mandibular branch of the trigeminal nerve, 


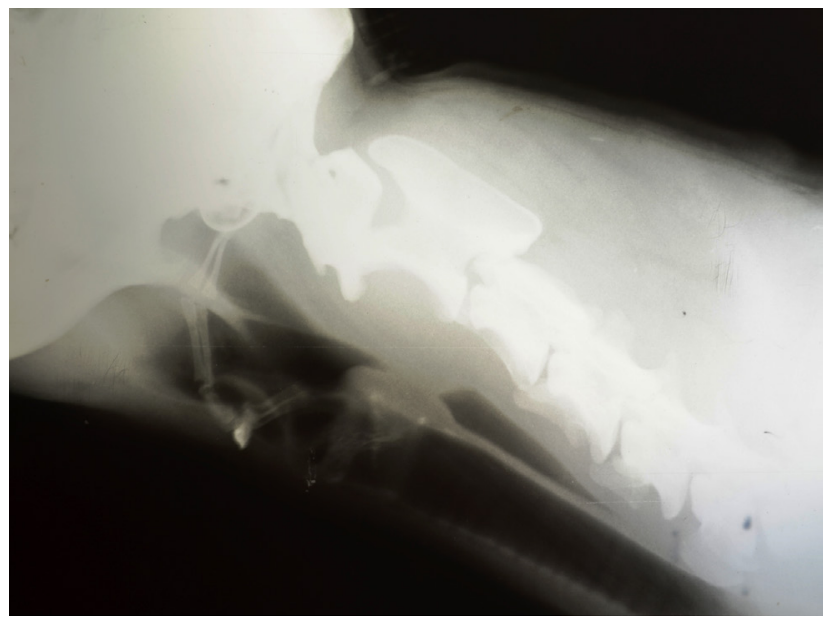

Figure 1. Lateral conventional radiograph of the cervical esophagus shows a radiopaque structure with soft tissue density dorsal to the cricoid cartilage and air in the cranial esophagus.

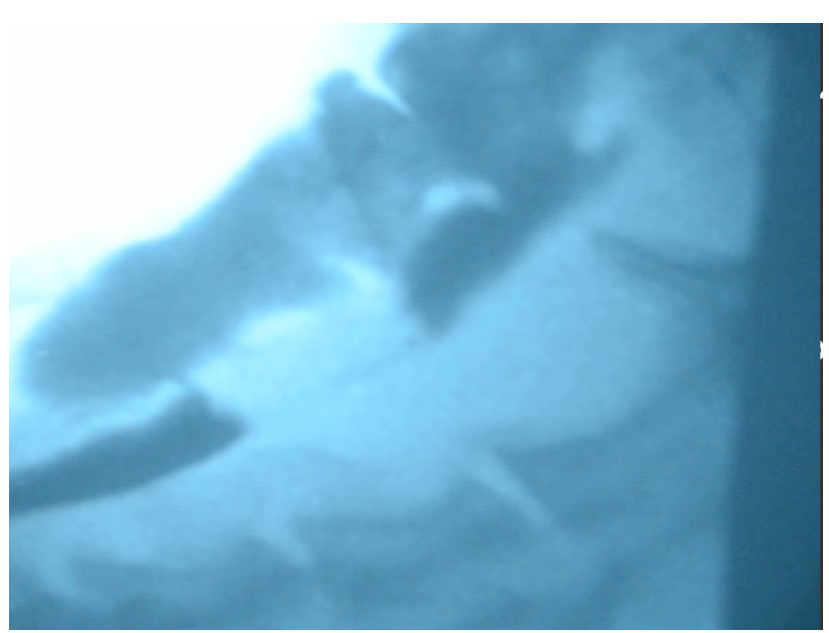

Figure 2. Esophageal fluoroscopy with iodinated contrast administered within food. The image shows a soft tissue density structure at the ventral wall of the esophagus. Additionally, contrast fluid was observed into the trachea.

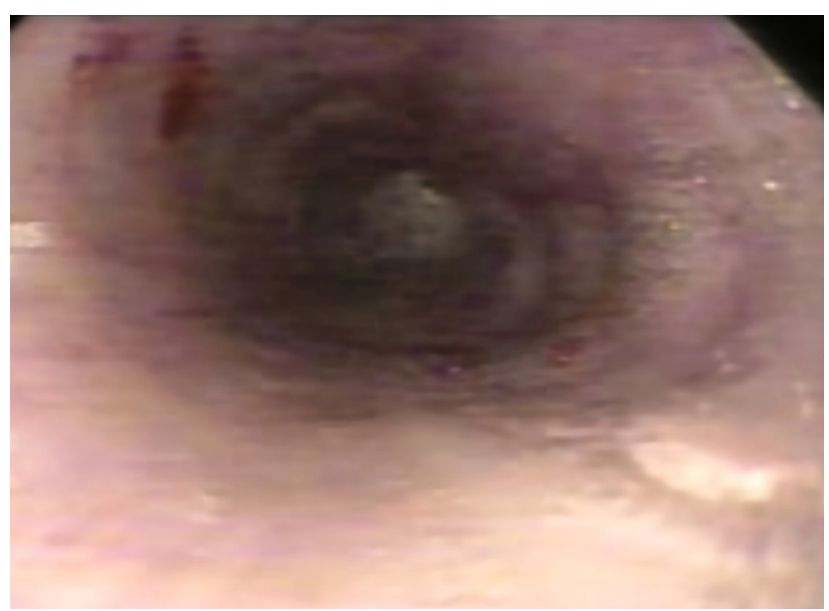

Figure 3. Tracheoscopy showed a hyperemic trachea with hemorrhagic areas and abundant mucus. including the masseter, temporalis, pterygoids, tensor tympani, and tensor veli palatini muscles [6].

Seven days after doxycycline treatment the dog showed a slight improvement, with a better appetite and increased weight. On physical examination, the dropping jaw was no longer apparent. However, the dysphagia continued. Preoperative laboratory analyses were repeated, and all values were within the normal ranges. Therefore, the animal underwent endoscopy. Tracheoscopy and bronchoscopy were performed first. The walls of the trachea and bronchi were hyperemic with hemorrhagic areas and abundant mucus. Bronchial washing and brushing was performed for cytological examination (Figure 3).

Next, esophagoscopy was performed, and a mass with smooth, regular borders was observed in the ventral wall of the esophagus. Because of the hard consistency of the mass, it was impossible to obtain a biopsy sample, but a cytology specimen was obtained via brushing. The endoscopic image was suggestive of an S. lupi nodule, therefore, a fecal analysis was indicated.

A severe suppurative inflammatory reaction was reported on the cytologic analysis of the tracheobronchial washing and brushing studies. No significant changes were found from the esophageal brushing samples. Fecal analysis revealed embryonated, thickshelled, small parasitic ova of S. lupi. Hence the case was diagnosed as spirocercosis.

Treatment with milbemycin oxime (Interceptor $\left.{ }^{\circledR}\right)^{5}$ was started at a dose of $0.5 \mathrm{mg} / \mathrm{kg}$ PO SID administered on days 0,7 , and 27 , followed by administration once a month for six months.

The dog went home on treatment for ehrlichiosis and spirocercosis, and a feeding regime similar to that for megaesophagus with metabolic energy requirements diet for weight gain.

One month after initiating treatment, the dog no longer exhibited regurgitation. The dog was fed similar to a megaesophagus patient without restriction, 4-5 times a day with a homemade diet. At physical examination the patient was normal and had gained weight.

Three months after treatment with milbemycin oxime the dog showed significant improvement, had no further dysphagia, regurgitation, or cough and reached $26 \mathrm{~kg}$ in body weight. Six months after starting treatment the patient was eating a commercial dry food in the normal position and continued to be free of clinical signs. 


\section{DISCUSSION}

The German shepherd dog has been reported to be a high risk for Spirocerca lupi infection [20,22]. The animal in this case presented some characteristic signs of spirocercosis such as regurgitation, vomiting, weight loss, coughing and hypochromic microcytic anemia $[3,13,17]$; however, the dog presented chronic dysphagia as the principal sign, which is not commonly reported for this disease.

Dysphagia in dogs is classified based on the location of the abnormality and this can be divided into oropharyngeal, esophageal and gastroesophageal [18]. Esophageal masses may result in dysphagia, and in particular, those involving the thoracic esophagus can be identified radiographically. Spirocerca lupi nodules can result in radiographically apparent esophageal masses. However most nodules produced by this nematode are present in the thoracic esophagus near the lower esophageal sphincter [3]. Due to this common location, dysphagia is not reported as a common sign in dogs with $S$. lupi [5,23], and in cases where it does happen, dysphagia would be classified such gastroesophageal type. In contrast, the cranial esophageal nodule that presented in this dog caused an esophageal dysphagia. Nodules resulting from $S$. lupi can protrude into the esophageal lumen in cases of early to mature nodules. Usually there are one to four nodules in the caudal esophagus submucosa ranging from $<1$ to $>4 \mathrm{~cm}$ in diameter that contain between three and six worms [2]. The nodules vary and not only bulge into the lumen of the esophagus but also distort the esophageal wall and extend into the surrounding mediastinal tissues [22]. Esophagoscopy is reported to be the most sensitive method for spirocercosis diagnosis [19]. Benign nodules are usually described as small and smooth with a nipple-like protuberance. In contrast, neoplastic nodules generally are roughened, ulcerated and present a necrotic surface that should be biopsied [3]. However, in a report of two cases of spirocercosis the nodules were described as smooth, which was an unexpected finding in malignant nodules [5]. Even though endoscopy is a reliable tool, unclear cases need to be monitored carefully for their response to treatment.

In the present case the nodule was relatively large (approximately $4 \mathrm{~cm}$ ) and had a smooth appearance with a small opening. However, the nodule had a hard consistency which prevented us from obtaining a biopsy with $2.3 \mathrm{~mm}$ endoscopic forceps. We think a factor for failing to obtain the sample was the cranial location of the nodule, because it was mentioned that the distal portion of the esophagus, just at the gastroesophageal junction, is the most feasible zone to obtain endoscopic biopsies. However, even in this distal area, biopsies are not always obtained or are of diagnostic quality, because only $40 \%$ of biopsies from healthy dogs are of diagnostic quality $[15,16]$. The strength of the stratified squamous epithelium over intact esophageal mucosa supports the resistance to damage created by endoscopic biopsy forceps. Successive biopsies at the same site for deep tissue of the lamina propria are recommended to maximize diagnostic information [18,22].

In this case, a tracheal brush sample demonstrated a severe suppurative inflammatory reaction secondary to aspiration pneumonia [7] which could explain the chronic cough. However, it is also possible that the growth of the nodule in the wall of the esophagus may have pressed on the trachea causing coughing.

The mandibular paralysis in this animal was initially attributed to a polymyositis secondary to $E h$ rlichia canis which can affect the muscles innervated by the mandibular branch of the trigeminal nerve [6]. However, one week after treatment with doxycycline the patient's jaw dropping improved but the dysphagia continued. It was therefore likely that the dysphagia was not secondary to polymyositis and the dropped jaw could have been due to canine trigeminal neuropathy which improves without treatment after an average of 22 days [14].

Moreover, although the normochromic normocytic anemia was reported as due to spirocercosis, in our case it could also have been secondary to ehrlichiosis because after treatment with doxycycline the blood analysis was normal [11].

Some drugs have been used for the treatment of spirocercosis in dogs [12]. For example, doramectin has been effective; however, administration is inconvenient as it has be to injected [17]. Milbemycin oxime administered orally is a relatively inexpensive and efficacious drug against $S$. lupi in dogs. The treatment was successful in animals with nodules and is effective in resolving clinical signs. In this case, esophagoscopy studies must be repeated to evaluate the evolution of the nodule and the effectiveness of treatment, which according to case reports, may take 3-5 months to clear the spirocercosis nodule $[8,9,17]$. 


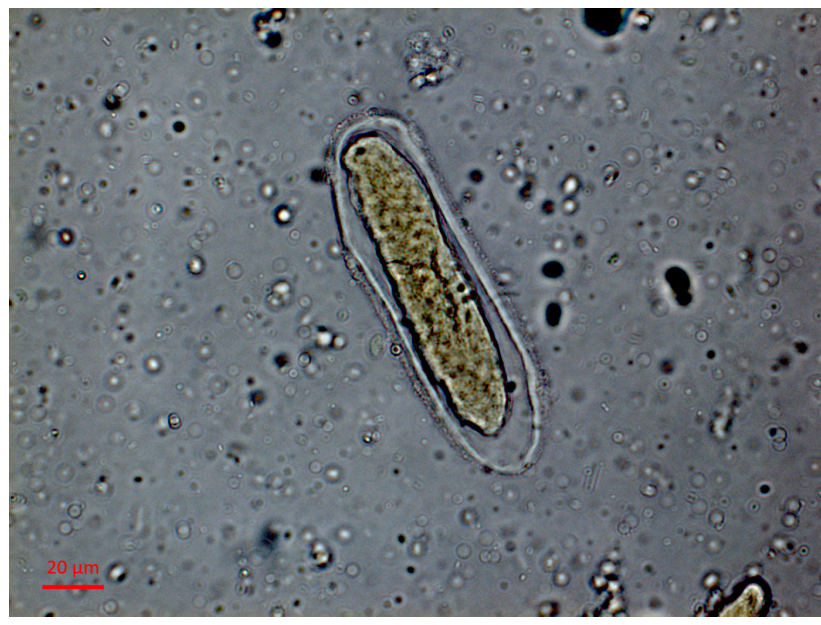

Figure 4. Fecal analysis showed embryonated, thick-shelled, small parasitic ova of Spirocerca lupi.

The present case is the first official report of the presence of canine spirocercosis in Ciudad Juarez, Mexico. It describes a German shepherd dog with an esophageal nodule secondary to $S$. lupi whose main sign was chronic dysphagia. Although dysphagia is reported as a sign of spirocercosis in dogs, rarely is it the most important sign as in this case. The dysphagia in this dog was attributed to the nodule location in the cranial esophagus, which is also rare. Endoscopy is an effective diagnostic modality, even if biopsies are not obtained. If suggestive nodules are noted in the esophagus, serial fecal analyses are required for a definitive diagnosis. The patient presented in this case improved with medical treatment with milbemycin oxime; the dog no longer shows dysphagia or odynophagia, has stopped coughing, has an improved body condition and is more active. In dogs, even though prevalence is low, Spirocerca lupi nodules should therefore be considered as a cause of dysphagia.

\section{MANUFACTURERS}

${ }^{1}$ Boehringer Ingelheim. Guadalajara, Jal, México.

${ }^{2} \mathrm{PiSA}$, Farmaceutica Mexicana. Guadalajara, Jal, México. ${ }^{3}$ IDEXX Laboratories. Westbrook, ME, USA

${ }^{5}$ Pfizer S.A. de C.V. Toluca, Edo. de México, México.

${ }^{6}$ Novartis Animal Health. Greensboro, NC, USA.

Acknowledgments. The videoendoscopy was purchased with funds FOMIX-CONACYT. The microscope was purchased with funds CONACYT. The authors would like to thank to all residents of the Veterinary Teaching Hospital. Also, they wish to thank to MC José Manuel Martínez for his help in editing pictures.

Declaration of interest. The authors report no conflicts of interest. The authors alone are responsible for the content and writing of the paper.

\section{REFERENCES}

1 Austin C.M., Kok D.J., Crafford D. \& Schaper R. 2013. The efficacy of a topically applied imidacloprid 10\%/moxidectin $2.5 \%$ formulation (Advocate ${ }^{\circledR}$, Advantage ${ }^{\circledR}$ Multi, Bayer) against immature and adult Spirocerca lupi worms in experimentally infected dogs. Parasitology Research. 112: 91-108.

2 Bailey W.S., Cabrera D.J. \& Diamond D.L. 1963. Beetles of the family Scarabaeidae as intermediate hosts for Spirocerca lupi. The Journal of Parasitology. 49: 485-488.

3 Christie J., Schwan E.V., Bodenstein L.L., Sommerville J.E.M. \& van Merwe LL. 2011. The sensitivity of direct faecal examination, direct faecal flotation, modified centrifugal faecal flotation and centrifugal sedimentation/flotation in the diagnosis of canine spirocercosis. Journal of the South African Veterinary Association. 82(2): 71-75.

4 Dvir E., Perl S., Loeb E., Shklar-Hirsch S., Chai O., Mazaki-Tovi M., Aroch I. \& Shamir M.H. 2007. Spinal intramedullary aberrant Spirocerca lupi migration in 3 dogs. Journal of Veterinary Internal Medicine. 21(4): 860-864.

5 Dvir E., Kirberger R.M. \& Malleczek D. 2001. Radiographic and computed tomographic changes and clinical presentation of spirocercosis in the dog. Veterinary Radiology and Ultrasound. 42: 119-129.

6 Evans J., Levesque D. \& Shelton D. 2004. Canine inflammatory myopathies: A clinic-pathological review of 200 cases. Journal of Veterinary Internal Medicine. 18: 679-691.

7 Hawkins E.C. 2014. Respiratory System Disorders. In: Nelson R.W. \& Couto C.G. (Eds). Small Animal Internal Medicine. 5th edn. St. Louis: Elsevier-Mosby, pp.217-366.

8 Kelly P., Fisher M., Lucas H. \& Krecek R. 2008. Treatment of esophageal spirocercosis with milbemycin oxime. Veterinary Parasitology. 156(3): 358-60.

9 Kok D., Williams E., Schenker R., Archer N. \& Horak I. 2010. The use of milbemycin oxime in a prophylactic anthelmintic programme to protect puppies, raised in an endemic area, against infection with Spirocerca lupi. Veterinary Parasitology. 174(3): 277-284.

10 Laflamme D. 1997. Development and validation of a body condition score system for dogs. Journal of Canine Practice. 
22: 10-15.

11 Lapin M.R. 2014. Infectious Disease. In: Nelson R.W. \& Couto C.G. (Eds). Small Animal Internal Medicine. 5th edn. St. Louis: Elsevier-Mosby, pp.1283-1399.

12 Lobetti R.G. 2000. Survey of the incidence, diagnosis, clinical manifestations and treatment of Spirocerca lupi in South Africa. Journal of the South African Veterinary Association. 71: 43-46.

13 Londoño Y., Carmona J.U. \& Giraldo C.E. 2009. Osteosarcoma generalizado y megaesófago secundario, ocasionados por infección de Spirocerca lupi en un canino. Revista Colombiana de Ciencias Pecuarias. 16(1): 63-69.

14 Mayhew P.D., Bush W.W. \& Glass E.N. 2002. Trigeminal neuropathy in dogs: a retrospective study of 29 cases (19912000). Journal of the American Animal Hospital Association. 38(3): 262-270.

15 Münster M., Bilzer T., Dettmann K., Hörauf A. \& Vieth M. 2012. Assessment of the histological quality of endoscopic biopsies obtained from the canine gastro-esophageal junction. Tierärztliche Praxis Kleintiere. 40(5): 318-324.

16 Münster M., Vieth M., \& Hörauf A. 2013. Evaluation of the quality of endoscopically obtained esophageal biopsies in the dog. Tierärztliche Praxis Kleintiere. 41(6): 375-382.

17 Okanishi H., Matsumoto J., Hiromi A., Kagawa Y., Asano K., Nogami S. \& Watari T. 2013. Successful resolution of esophageal nodules in a dog infected with Spirocerca lupi. The Journal of Veterinary Medical Science. 75(12): 1629-1632.

18Pollard R.E. 2012. Imaging Evaluation of dogs and cats with dysphagia. ISRN Veterinary Science. 1-15. doi:10.5402/2012/238505

19 Ranen E., Lavy E., Aizenberg I., Perl S. \& Harrus S. 2004. Spirocercosis-associated esophageal sarcomas in dogs. A retrospective study of 17 cases (1997-2003). Veterinary Parasitology. 119: 209-221.

20 Sanghai A.A., Bhojne G.R. \& Dakshinkar N.P. 2015. Successful management of spirocercosis in a dog. Indian Journal of Canine Practice. 7(2): 130-131.

21 Sheta E., Elzomor S., Farghali H., Ahmed K.A. \& Elkader N.A.A. 2014. Endoscopic depiction and treatment evaluation of Spirocerca lupi in dogs. Global Veterinaria. 13(2): 258-265.

22 Van der Merwe L.L., Kirberger R.M., Clift S., Williams M., Keller N. \& Naidoo V. 2008. Spirocerca lupi infection in the dog: a review. The Veterinary Journal. 176(3): 294-309.

23 Willard M.D. 2014. Digestive System Disorders. In: Nelson R.W. \& Couto C.G. (Eds). Small Animal Internal Medicine. 5th edn. St. Louis: Elsevier-Mosby, pp.367-500.

24 Yas E., Kelmer G., Shipov A., Ben-Oz J. \& Segev G. 2013. Successful transendoscopic oesophageal mass ablation in two dogs with Spirocerca lupi associated oesophageal sarcoma. Journal of Small Animal Practice. 54(9): 495-498. 